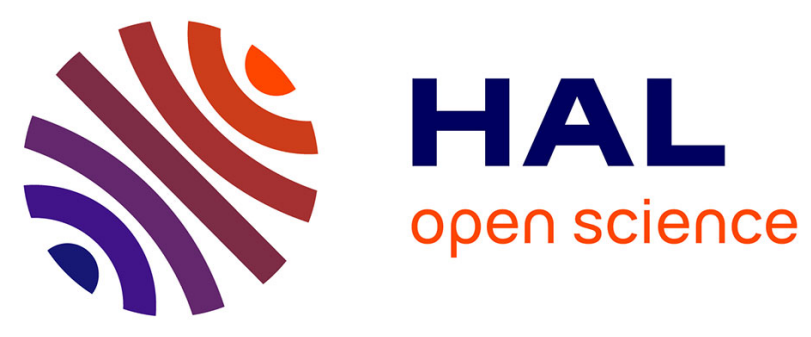

\title{
Synthesis of an electric sensor based control for underwater multi-agents navigation in a file
}

Mohammed-Rédha Benachenhou, Christine Chevallereau, Vincent Lebastard, Frédéric Boyer

\section{- To cite this version:}

Mohammed-Rédha Benachenhou, Christine Chevallereau, Vincent Lebastard, Frédéric Boyer. Synthesis of an electric sensor based control for underwater multi-agents navigation in a file. 2013 IEEE International Conference on Robotics and Automation (ICRA), May 2013, Karlsruhe, Germany. pp.4608 - 4613, 10.1109/ICRA.2013.6631232 . hal-00994947

\section{HAL Id: hal-00994947 \\ https://hal.science/hal-00994947}

Submitted on 22 May 2014

HAL is a multi-disciplinary open access archive for the deposit and dissemination of scientific research documents, whether they are published or not. The documents may come from teaching and research institutions in France or abroad, or from public or private research centers.
L'archive ouverte pluridisciplinaire HAL, est destinée au dépôt et à la diffusion de documents scientifiques de niveau recherche, publiés ou non, émanant des établissements d'enseignement et de recherche français ou étrangers, des laboratoires publics ou privés. 


\title{
Synthesis of an electric sensor based control for underwater multi-agents navigation in a file
}

\author{
M-R. Benachenhou, C. Chevallereau, V. Lebastard, F. Boyer,
}

\begin{abstract}
Thanks to an electro-sensible skin, some species of fish can feel the surrounding electric field generated by them-self or other fish. Known under the name of "electricsense", this ability allows these fish to navigate in confined surroundings. Based on a bio-inspired electric sensor, this article presents how this electric sense can be used for the navigation in formation of several underwater vehicles. The formation considered is a file, each vehicle is assumed to follow its predecessor at a given distance. In confined environment, the file formation is interesting since fish can follow the same safe path. Being based on the servoing of the electric measurements, these laws do not require the knowledge of the location of the agents. The underwater vehicle studied have non holonomic properties, their forward velocity has no lateral component. Depending on the choice of the controlled outputs (combination of electric measures) we will see that path followed by the follower agents can be different and a methodology to choose the output will be defined in order that all the agents follow the leader path in presence of curved motion of the leader. The influence of the number of electrodes is discussed. Simulation results illustrate the proposed approach.
\end{abstract}

\section{INTRODUCTION}

Electric sense is a means of perception used by some fish living in tropical forests of Africa and South-America [2]. In the fish of the mormyrides family, the polarization of an Electric Organ of Discharge located in its tail generates a dipolar electric field around the fish. Electric fish used a passive version of the electric sense [3] in which the electric field is generated by exogenous sources, as other electric fish nearby, since these fishes swim in groups for most of time. Biological experiments have revealed that electric fish use specific strategies to organize their collective electric activity [4]. In particular, in order to avoid jamming and overlapping between individual electric pulses, a group of mormyride orders the electric activity of each member of the group in a fixed sequence of individual pulses separated by "silent periods".

The objective of this article is to explore the possibilities offered by electric senses in order to address the problem of navigation of a group of agents moving in a file. In order to achieve this goal, we use a sensor recently proposed in [1]. This active sensor is a slender shape insulating shell on which are fixed two hemispherical tip electrodes as well as a set of intermediate annular ones (see figure 1). We

This work has been supported by the ANGELS project funded by the European Commission, Information Society and Media, Future and Emerging Technologies (FET) contract number: 231845

the authors are with CNRS, Ecole des Mines de Nantes, Irccyn, Nantes, France, (Christine.Chevallereau, Frederic.Boyer, Vincent.Lebastard,

Mohammed-Redha. Benachenhoudirccyn.ec-nantes.fr) will consider here a group of these rigid slender robots (or agents) moving in a common horizontal plane. The motion of each module is controlled through its axial velocity (aligned with the probe axis) and its vertical angular velocity using the kinematic model of a (non-holonomic) unicycle, since the robot (and the fish) cannot move laterally (see figure 1). In a formation, the agents will have to keep a specified

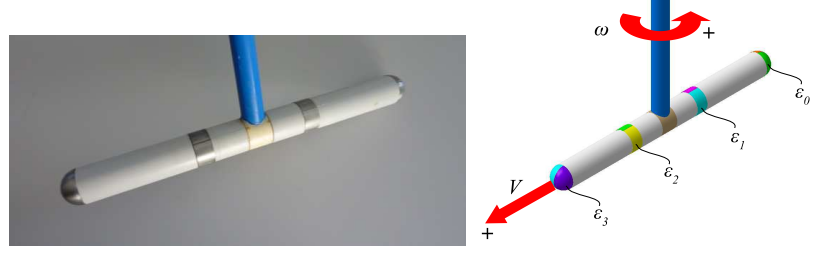

Fig. 1. Picture (left) and schematic view (right) of a 4-electrode sensor.

relative position and orientation between them. In order to control the relative position of a passive agent with respect to the active one; we could first seek to invert the model of the passive agent's electric measurements with respect to the position and orientation between the two agents [5]. Unfortunately, this problem is difficult [6], a more direct approach, a sensor-based control approach [7], [8], [9] based on a direct feedbacks from the measurements is used here. This approach can be named "electric sense servo control" for underwater navigation. The passive agent senses the electric field and seeks some prescribed measurements ensuring him a specified relative configuration. The principle of this approach and stability of formation have been presented in [10]. In this paper, the performance of the control is considered and a methodology is proposed to determine the appropriate synthesis of the control based on a desired behaviour for a file of agents. Our objective is that the agents follow the same path than the leader even in presence of turn of the leader. The effect of the number of electrodes onto the path followed by the agents is also discussed.

The article is structured as follows. In section II, the nonholonomic model of motion of the vehicle are recalled and the desired behaviour of the file formation is defined. In III we present the model of the electric sense as well as the available measurements. In section IV, the principles of "electric sense servo control" is presented and the behaviour of the agent in closed loop is presented. In section $\mathrm{V}$, an original methodology to synthesize the control law based on the desired behaviour in closed loop is proposed. In section VI, some simulation examples illustrate the impact on the number of electrodes on the performance obtained. 


\section{MOTION IN FORMATION OF A SET OF NON-HOLONOMIC ROBOT}

The robots move in a common horizontal plane. The motion on the vertical direction (buoyancy) is controlled independently based on pressure sensor and change on the volume of the robot. To approximate the motions of a swimming fish, the robots' motions are modelled using the non-holonomic kinematic model of the unicycle. The control inputs are the linear axial velocity $V$ and the angular vertical velocity $\omega$ (figures 1 ). When the robots are controlled in formation, the objective is of keeping a constant position and orientation (or "situation") between the robots. Following a frequently used strategy in multi-robot navigation [11], one of the robots is distinguished as a "leader" defining a reference motion for the group. This reference motion is unknown to the other robots, or "followers", and is compatible with the non-holonomic constraints.

Combining the "follower-leader" and the "active-passive" strategies, in a first step, the active agent is the leader and its closest neighbour is passive, it follows the leader using the sensor-based control. In the second step, this following agent then becomes active and its neighbour follows it using the sensor-based control. This principle is propagated from one agent to the other looping in an ordered sequence so that the reference motion is propagated to the whole group incrementally. For the sake of clarity, in the following description, we will only consider the two first agents.

\section{A. The motion model}

The leader velocity is defined by $\left(V_{r}, \omega_{r}\right)$. The velocity $V$ and $\omega$ of the passive agent are the control inputs $u$ of the problem: $u=(V, \omega)^{T}$. The situation of the passive agent with respect to the active agent's frame is defined by a state vector $X=[x, y, \theta]^{T}$. The control objective is to maintain $X$ at a desired value $X^{d}=\left[0, y^{d}, 0\right]^{T}$ for a file formation where $y^{d}$ is the distance between the centre of two following agents. Our control model is defined by $\dot{X}$ expressed as a function of the control inputs $V, \omega$ of the passive agent. This model is [10]:

$$
\dot{X}=J u+P(t)=\left[\begin{array}{cc}
-\sin (\theta) & 0 \\
\cos (\theta) & 0 \\
0 & 1
\end{array}\right] u+\left[\begin{array}{c}
y \omega_{r} \\
-V_{r}-x \omega_{r} \\
-\omega_{r}
\end{array}\right]
$$

where $P(t)$ is a bounded perturbation due to the unknown motion of the active agent. The behaviour of the follower robot being governed by (1), the combination of the two first equations allows writing a relation between the state $X$, its time derivative $\dot{X}$, and the reference motion of the active agent, independent of the control input $u$ :

$\dot{x} \cos (\theta)+\dot{y} \sin (\theta)=-V_{r} \sin (\theta)+y \omega_{r} \cos (\theta)-x \omega_{r} \sin (\theta)$.

This equation reflects the non holonomic constraints for tracking of the leader whose velocity is $\omega_{r}, V_{r}$.

\section{B. Desired behaviour for a file formation}

For a turn of the leader, the desired relative situation $X^{d}$ between leader and follower cannot be achieved (see equation (2)). But in the case of a turn of the leader what is the appropriate motion of the follower? To avoid large displacement of the follower, it seems that the more elegant behaviour of the follower is to follow the same path as the leader. Moreover, since all the robots have the same type of actuator, if the follower is initially on the leader path, it can continue to follow the leader for any admissible path of the leader while it is not able to conserve a constant relative situation. In the case of a motion of a leader with a curvature $\omega_{r} \neq 0$, the desired equilibrium situation of the follower is presented in figure 2. The idea is that the follower must be on the circle currently followed by the leader and that the desired distance between the two agents $y^{d}=-d$ is the arc-length.

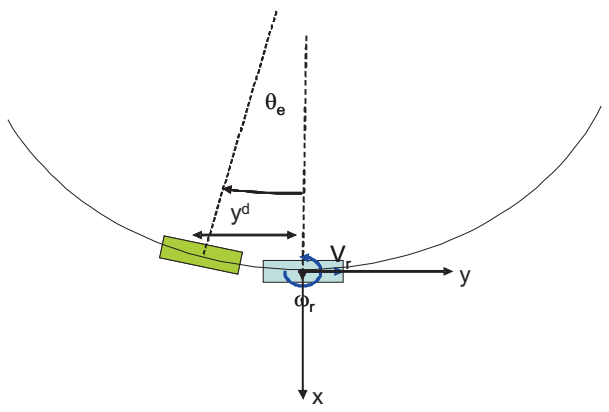

Fig. 2. Based on the current curvature of the leader, the circle that the leader follows is defined. The desired equilibrium position for the follower is on this circle at an arc-length distance $d$.

Based on figure 2, the desired relative situation is:

$$
\begin{aligned}
& \theta_{e}=-\frac{d}{R} \\
& x_{e}=-R\left(1-\cos \left(\theta_{e}\right)\right) \\
& y_{e}=R \sin \left(\theta_{e}\right)
\end{aligned}
$$

where $R$ is the curvature of the leader turn, $R=\frac{V_{r}}{\omega_{r}}$.

This desired behaviour must be obtained for any curvature $R$. Reporting the first equation of (3) in the two last equations we obtained as set of desired relative position, for $\theta_{e} \neq 0$ :

$$
\begin{aligned}
& x_{e}=\frac{d}{\theta_{e}}\left(1-\cos \left(\theta_{e}\right)\right) \\
& y_{e}=-\frac{d}{\theta_{e}} \sin \left(\theta_{e}\right)
\end{aligned}
$$

For $\theta_{e}=0$, the corresponding state is $x_{e}=0, y_{e}=$ $-d$. It can be noted that the state satisfying this condition corresponds to the desired relative situation if we have also $\theta_{e}=-\frac{d \omega_{r}}{V_{r}}$. Considering the non holonomic constraints, our control objective is rewritten as: the relative situation of the follower with respect to the leader must be $X=X_{e}$ where $X_{e}=\left[x_{e}, y_{e}, \theta_{e}\right]$ satisfy (3).

\section{ELECTRIC SENSE, SENSOR AND MEASURES}

The sensors (that we will also name "agents" or "robots") are slender probes composed of $N$ electrodes linked by insulating axi-symmetric boundaries (see figure 1). Each sensor has two working modes [1]. In the first mode, the 
two tip electrodes are electrically connected by a voltage generator (AC source) which imposes a controlled voltage between them, while all the other electrodes are disconnected. This mode is said "active", since once immersed in a conductive fluid, the probe generates a dipolar electric field in its surroundings. In the second mode, all the electrodes are electrically disconnected from each other and the probe measures the electric potential produced by an exogenous electric field on each of its electrodes with respect to a common ground. This working mode is said "passive". In order to avoid jamming between several agents' electric fields, only one agent is active while all the others are passive. The change between passive and active mode is instantaneous and periodic.

Due to the slender geometry, the potential generated by any active agent can be expressed as [12]:

$$
\phi(\mathbf{x})=\frac{I}{4 \pi \gamma}\left(\frac{1}{r_{h}}-\frac{1}{r_{t}}\right) .
$$

where $\gamma$ the conductivity of the ambient fluid, $I$ the total currents flowing across the two tip electrodes. Equation (5) is a leading order approximation of the potential with respect to the small quantity $R / l \simeq R / r_{h} \simeq R / r_{t}$ where $R, l$, $r_{h}$ and $r_{t}$ respectively denote the radius of the cylindrical probe, its length (between its two tip electrodes) and the distance from the head and the tail electrodes to the point $\mathbf{x}$ at which the potential is evaluated. The potential field

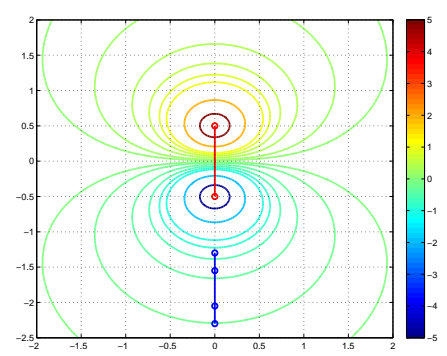

Fig. 3. The active dipole emits a dipolar potential field, which can be measured by the electrodes of the passive agent.

produced by the active agent is represented in the figure 3, in the case $\frac{I}{4 \pi \gamma}=1$ and $l=1 \mathrm{~m}$. Now, let us consider an agent in passive mode. The electrodes are small enough to provide a point measurement of the potential field (produced by the active agent) without perturbing it. Only the voltage difference can be measured, so for a passive agent with $N$ electrodes, we define $N-1$ measurements of difference of voltage: $U_{i}=\phi_{i}-\phi_{1}$ for $i=2, . . N$. Furthermore, each of the electrodes $\mathcal{E}_{i}$ is divided into two identical left and right independent sub-electrodes, on which the electric voltage can be measured (see figure 1). Averaging the left and right voltages, the voltage $U_{i}$ at the centre of the electrodes $\mathcal{E}_{i}$ is evaluated. Due to the slenderness of the agent, the lateral (left minus right) difference of voltages is a direct image of the lateral electric field $E_{i}$ at the centre of each of the $\mathcal{E}_{i}$. The measurements can be written as: $m=m(X)$, where $X=[x, y, \theta]^{T}$ and $m=\left[U_{2}, \ldots, U_{N}, E_{1}, \ldots, E_{N}\right]^{T}$, the number of measurements is $n_{m}=2 N-1$.

\section{ELECTRIC SENSE SERVO CONTROL}

\section{A. The control law}

Because the motion of the passive agent is defined by two inputs $V$ and $\omega$, only two outputs can be tracked in closed loop. The control law is based on the measurements produced by the electric sensor with no calculation of the relative situation of the leader and follower agents. The two controlled outputs are two linear functions of the electric measurements $s=C m(X(t))$ where $C$ is a constant $(2 \times(2 N-1))$ matrix and $m(X(t))$ denotes the vector of measurements. The corresponding desired value is $s^{d}=C m\left(X^{d}\right)$, and the output vector of error $e(t)$ is:

$$
e(t)=C m(X(t))-s^{d}
$$

By differentiating this equation, and using (1), the control input appears. Since the desired relative configuration, is fixed, $s^{d}$ is constant and we have:

$$
\dot{e}(t)=C \frac{\partial m}{\partial X}(J u+P(t)) .
$$

The objective of the control law, is to zeroed $e(t)$, the following control law, $0<\lambda<1$ :

$$
u=-\lambda L e=-\lambda\left(C \frac{\partial m \widehat{\left(X^{d}\right)}}{\partial X} J\left(X^{d}\right)\right)^{-1} e
$$

is proposed to achieve this objective, where $L$ is an estimation of $\left(C \frac{\partial m(X)}{\partial X} J(X)\right)^{-1}$ at the desired state. If the active robot is at rest $(P(t)=0)$, the convergence condition that ensures the decrease of the error is given by [8]:

$$
\left(C \frac{\partial m}{\partial X} J\right) L>0
$$

Thus, the matrix $L$ defined in (8) is the best choice when the control has converged. In transient phase $\left(X \neq X^{d}\right)$, the condition (9) must be checked. A condition on the choice of the controlled output (i.e. on $C$ ) consists of ensuring that $\left(C \frac{\partial m}{\partial X}\left(X^{d}\right) J\left(X^{d}\right)\right)$ is not singular. This condition is not difficult to achieve and is checked when $C$ is selected. For all the cases tested in section VI, the condition (9) is satisfied. The perturbation $P(t)$ due to the motion of the active agent induces a bounded error $e(t)$.

\section{B. The closed-loop behaviour of the agents}

Since the relative situation of the agents are defined by the three variables $x, y, \theta$, an infinite set of relative situations that will produce the desired outputs $C m(X)=s^{d}$ exists. This means that the control law (8) that insures that $C m(X)=s^{d}$ does not guaranties that $X=X^{d}$. The closed loop dynamics of the system when the output errors are forced to zero (called "zero dynamics" [13]) must be studied. In particular, the control outputs must be chosen in order to produce, at least locally, a stable zero dynamics.

The zero dynamics characterizes the displacement of the robot when the controlled outputs are identically zero. In this case, the state of the robot remains in $Z=$ $\left\{X \mid C m(X)=s^{d}\right\}$. Since the dimension of $Z$ is 1 , around 
$X^{d}$, the state $X$ belonging to $Z$ can be parameterized by 1 variable. We assume that $\theta$ is monotonic in $Z$ around $\theta=\theta^{d}=0$ and thus $\theta$ is chosen to parameterize $Z . X \in Z$ can be written as: $\left[x^{Z}(\theta), y^{Z}(\theta), \theta\right]^{T}$. In closed loop, the state of the robot remains on $Z$, thus its velocity $\dot{X}$ satisfies:

$$
\left[\begin{array}{c}
\dot{x} \\
\dot{y}
\end{array}\right]=\left[\begin{array}{c}
\frac{\partial x^{Z}(\theta)}{\partial \theta} \\
\frac{\partial y^{Z}(\theta)}{\partial \theta}
\end{array}\right] \dot{\theta}=\left[\begin{array}{c}
Z_{x}(\theta) \\
Z_{y}(\theta)
\end{array}\right] \dot{\theta},
$$

In fact, being a non holonomic system, the situation of the follower robot is governed by (2) for any motion of the leader. In closed loop this equation must be combined with (10), to describe the behaviour of the follower agent as a function of $\theta$ and $\dot{\theta}$ :

$$
\begin{gathered}
Z_{x}(\theta) \dot{\theta} \cos (\theta)+Z_{y}(\theta) \dot{\theta} \sin (\theta)= \\
-V_{r} \sin (\theta)+y^{Z}(\theta) \omega_{r} \cos (\theta)-x^{Z}(\theta) \omega_{r} \sin (\theta) .
\end{gathered}
$$

This latter equation plays a crucial role, revealing the motion of the follower robot in closed loop.

\section{SyNTHESIS OF THE CONTROL LAW}

The question we have now to answer is whether the agent will be steered to the desired state $X=X_{e}$ satisfying (3), and if so will this equilibrium state be locally stable? The answer to these questions, obviously depend on the property of the zero dynamics. We will first show that the answer is yes for a particular choice of the zero dynamics manifold. And the section V-B, we will propose a methodology to defined to control outputs $C m(X)$ in order that closed loop zero dynamics be the expected one.

\section{A. The chosen zero dynamics manifold}

Based on the desired equilibrium state $X_{e}$, we chose zero dynamics manifold defined by:

$$
\begin{aligned}
& x^{Z}(\theta)=\frac{d}{\theta}(1-\cos (\theta)) \\
& y^{Z}(\theta)=-\frac{d}{\theta} \sin (\theta)
\end{aligned}
$$

and $x^{Z}(0)=0, y^{Z}(0)=-d$. For a desired distance between agents is $d=1.8 \mathrm{~m}$, the desired zero dynamics is represented in figure 4.

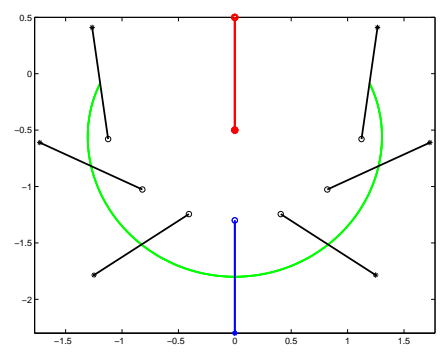

Fig. 4. Different locis of the passive agent configurations belonging to the desired zero dynamics defined by (12). The set of the sensor centre positions $x_{e}, y_{e}$ is represented by the green line. For certain situations, the sensor is drawn in black, and in blue for the desired situation. The active agent is drawn in red.

If we report the equation corresponding to this zero dynamics manifold in equation (11), we obtain for the closed loop behaviour of the robot:

$$
\dot{\theta}\left(\frac{d}{\theta^{2}}(1-\cos (\theta))\right)=-\left(V_{r}+\frac{d \omega_{r}}{\theta}\right) \sin (\theta) .
$$

This equation have two equilibrium solutions $(\dot{\theta}=0)$ corresponding to $\theta=0$ or $\theta=-\frac{d \omega_{r}}{V_{r}}=-\frac{d}{R}$ where $R$ is the curvature of the leader. Let us first consider the equilibrium which corresponds to $\theta=0$. The linearization of equation (13), gives:

$$
\dot{\theta} \frac{d}{2}=-V_{r} \theta-d \omega_{r}
$$

This equilibrium exist only for $\omega_{r}=0$ which corresponds to the straight line motion of the leader. If the passive agent follows the leader $\left(V_{r}>0\right.$ and $\left.d>0\right)$ then the zero dynamics is stable. Locally the passive agent will converges to the desired state $X^{d}=[0,-d, 0]$.

Now we will consider the case, $\omega_{r} \neq 0$, the leader turns, the equilibrium state of the follower is defined by $\theta_{e}=\theta=$ $-\frac{d}{R}$ and by (12), thus it is the desired equilibrium defined in equation (3). The linearization of equation (13), around $\theta_{e}=\theta=-\frac{d}{R}$ gives:

$$
\dot{\theta}=-V_{r} \frac{\theta_{e} \sin \left(\theta_{e}\right)}{d\left(1-\cos \left(\theta_{e}\right)\right)}\left(\theta-\theta_{e}\right)
$$

Assuming that the passive agent follows the leader $\left(V_{r}>0\right.$ and $d>0$ ), the zero dynamics will be locally stable for any value $-\pi<\theta_{e}<\pi$. As a conclusion if the outputs are defined such that the zero dynamics are defined by (4), the stability condition is satisfied and the follower follows the leader path for a motion of the leader with any curvature.

\section{B. Methodology to define the outputs}

Now we want to define the outputs to have such properties. It is possible to consider this problem as an optimisation problem where the optimisation variables is the matrix $C$ and the objective is to minimize the difference between the resulting zero dynamics and the desired zero dynamics. The optimisation process will require many calculations and a model of the sensor. To limit the calculation, we propose a different strategy that can be based either on a model of the sensor or on experimental data.

The desired zero dynamics has been defined, and is presented in figure 4 . The first step is to put the passive agent in a set of situations belonging to the desired zero dynamics and to record the measurements. The measurements obtained when the passive sensor of $1 \mathrm{~m}$ with 4 electrodes distributed along the sensor (see figure 3 ) evolves along the desired zero dynamics defined for $d=1.8$ is presented in figure 5. In fact we present the difference between the current measurements and the measurements corresponding to the desired configuration at $\theta_{e}=0: m\left(X_{e}\right)-m\left(X^{d}\right)$. The situation of the passive agent in the desired zero dynamics is parameterised by $\theta$.

If controlled outputs $C m(X)$ such that the zero dynamics is the desired one exist, then by definition $\operatorname{Cm}\left(X_{e}\right)-$ $C m\left(X^{d}\right)=0$, for any state $X_{e}$ belonging on the desired zero dynamics. The latter equation can be rewritten: 

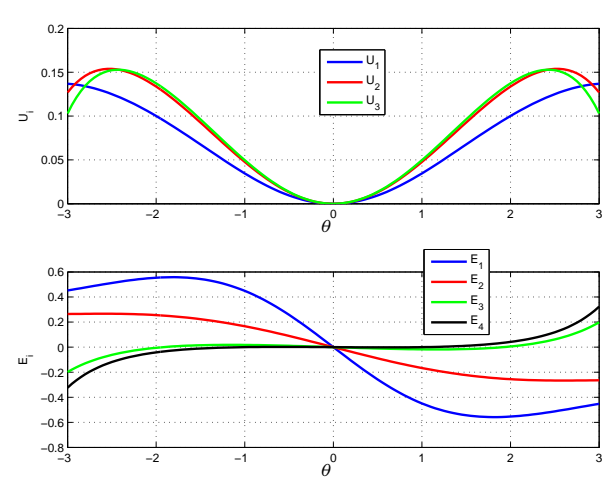

Fig. 5. The measurements $m\left(X_{e}\right)-m\left(X^{d}\right)$ recorded when the passive agent evolves along the desired zero dynamics for a sensor with 4 electrodes.

$$
C\left(m\left(X_{e}\left(\theta_{e}\right)\right)-m\left(X^{d}\right)\right)=0
$$

where $C$ is the $\left(2 \times n_{m}\right)$ matrix to be determined. This equation has to be satisfy for any value of $\theta_{e}$ (with $-\pi<$ $\theta_{e}<\pi$ ). $n_{s}$ samples of $X_{e}$ will be defined (with $n_{s}>>n_{m}$ ) and a concatenation of all the variations on measurements $m\left(X_{e}\left(\theta_{e}\right)\right)-m\left(X^{d}\right)$ is build column by column to define a matrix of measurements $M_{s}$. This $\left(n_{m} \times n_{s}\right)$ matrix can be constructed based on the model of electric sense or experimentally. According to (16), we want to have:

$$
C M_{s}=0
$$

If the rectangular matrix $M_{s}$ is full rank, then the only possibility is to have $C=0$. In order to be able to define a full rank matrix $C$ the rank of $M_{s}$ must be less than or equal to $n_{m}-2$. Using singular value decomposition (SVD), the matrix $M_{s}$ can be written as $M_{s}=U S V^{\prime}$ where $U$ and $V$ are orthogonal matrices, and $S$ is a rectangular $\left(n_{m} \times n_{s}\right)$ with on its diagonal positive term ordered in decreasing order. If the rank of $M_{s}$ is less than or equal to $n_{m}-2$, and the lines of $C$ is defined as the last two columns of $U$ then equation (17) is satisfied. The zero dynamics associated to $C$ coincides at the $n_{s}$ samples used to build $M_{s}$ with the desired zero dynamics. Since the rank of a matrix is defined numerically, the smallest singular values of $M_{s}$ defines how far the matrix $M_{s}$ is from being singular.

As a conclusion, the proposed methodology is the following. The two lines of $C$ are the last two columns of $U$ (matrix of the SVD decomposition of $M_{s}$ ). The two smallest singular values are an indicator on the proximity between the real zero dynamics and the expected one. Since, the controlled output are defined as a linear combination of the measurements available on the sensor, these two smallest singular values are probably not exactly zero, but small values indicate that the corresponding zero dynamics is close to the desired zero dynamics.

\section{Influence of the number of electrodes}

In the proposed methodology, the proximity of the zero dynamics and the desired one is quantified by the smallest eigenvalue of $M_{s}$ and depends obviously of the number

\begin{tabular}{|c|c|c|c|c|c|}
\hline \multirow{2}{*}{ nb elec. } & sensor & \multicolumn{4}{|c|}{ smallest eigenvalues } \\
\hline \hline 2 & $\mathrm{O}-1.8$ & \multicolumn{2}{c|}{$\mathrm{d}=1.3$} \\
\hline \multirow{3}{*}{3} & $\mathrm{O}-\mathrm{O}-\mathrm{O}-\mathrm{O}$ & 1.1107 & 0.09509 & 10.964 & 2.4016 \\
& $\mathrm{O}-\mathrm{O}-\mathrm{O}$ & 0.1347 & 0.1169 & 0.9586 & 0.1737 \\
& $\mathrm{O}-\mathrm{O}-\mathrm{O}$ & 0.0450 & 0.0096 & 0.0771 & 0.0480 \\
\hline \multirow{3}{*}{4} & $\mathrm{O}-\mathrm{O}-\mathrm{O}-\mathrm{O}$ & 0.0096 & 0.0012 & 0.0452 & 0.0193 \\
& $\mathrm{O}-\mathrm{O}-\mathrm{O}-\mathrm{O}$ & 0.0095 & 0.0012 & 0.0467 & 0.0041 \\
& $\mathrm{O}-\mathrm{O}-\mathrm{O}-\mathrm{O}$ & 0.0098 & 0.0007 & 0.0432 & 0.0342 \\
\hline 5 & $\mathrm{O}-\mathrm{O}-\mathrm{O}-\mathrm{O}-\mathrm{O}$ & 0.0007 & 0.0002 & 0.0028 & 0.0020 \\
\hline
\end{tabular}

TABLE I

THE SMALLEST SINGULAR VALUE OF $M_{S}$ FOR SEVERAL SETS OF ELECTROdes. SMALlER ARE THE SINGUlar VALUES, CLOSER IS THE PATH FOLLOWED BY THE AGENTS TO THE PATH OF THE LEADER.

of measurements and thus of the number of electrodes. Higher is the number of electrodes, closer can be the zero dynamics to the desired one, and better is the closed loop behaviour. These results are illustrated numerically in the table I. Different configurations of electrodes (numbers and positions) are chosen and the corresponding singular values are defined when the desired distance between the agents are respectively $1.8 \mathrm{~m}$ and $1.3 \mathrm{~m}$.

The length of the sensor is $1 \mathrm{~m}$, the head and tail electrodes are placed respectively at coordinates 0.5 and -0.5 . These two electrodes constitute the minimal set of electrodes. Up to three electrodes located at $-0.25,0$ or 0.25 can be added. The configuration considered is schematically represented in the second column of table I, the head of the sensor is on the left. It can be observed that when the number of electrodes varies from 2 to 5 , the smallest singular values decrease, the zero dynamics is closer to the desired one. This point is illustrated in simulation in the next section. When only two electrodes are used, since few measures are available, it is difficult to choose the shape of the zero dynamics. For 4 or 5 electrodes, since many measurements are available, the shape of the zero dynamics can approximated the desired one and the smallest singular values are small. For 3 electrodes, the results are influenced by the position of the intermediate electrode. Better result is obtained for an intermediate electrode closer to the tail. For different desired distance $d$ between agents, similar characteristics are obtained. Since for smaller distance, the amplitude of the measures is higher, the smallest singular values are higher.

\section{Simulation Results}

In this section, the closed loop behaviour of a file of 4 agents distant from $1.8 \mathrm{~m}$ between the centre of two following agents is simulated. The simulation is made using the kinematic model (1) and a fixed sampling period of $10 \mathrm{~ms}$, an initial error of $0.01 \mathrm{~m}$ in position and of $0.01 \mathrm{rd}$ in orientation between agents is introduced. For a same motion of the leader, electric model (section III) with 2 to 4 electrodes, are used. Even if stable behaviour can always be achieved, a larger number of available measurements allow us to have a better behaviour. We consider 3 cases 
corresponding to 2, 3, and 4 electrodes, and the line 1,4 and 5 of table I. During the first part the leader moves with constant linear velocity and increasing angular velocity and then the leader rotates with constant angular velocity. The constant linear velocity is $0.3 \mathrm{~m} / \mathrm{s}$. The results are illustrated on figure 6 with the paths followed by the four agents and by a final photo of the four agents for the 3 different sensors. In this latter graphs the circle followed by the leader is drawn in order to show if the follower agents are on this circle or not. A stable behaviour of the formation is observed in any case, but with 4 electrodes, the path followed by all the agents perfectly coincide (figure 6 (c) and (f)), this is not the case when only 2 electrodes are used (figure 6 (a) and (d)). With 3 electrodes the obtained behaviour is quite good (figure 6 (b) and (e)). A quantification of the quality of results is proposed in table II via the average distance between the path of the leader centre and of the $i^{\text {th }}$ agent centre ${ }^{1}$.
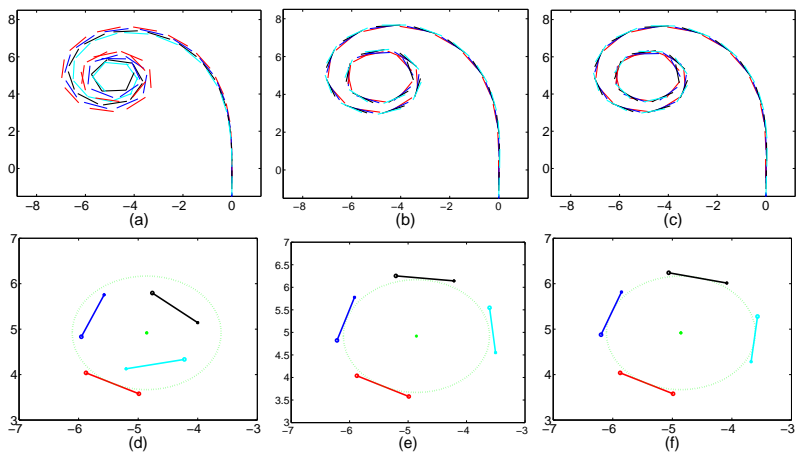

Fig. 6. a-c)The path followed by 4 agents equipped with 2,3 or 4 electrodes, the leader (red) has a complex path with varying curvature. d-f)The photo of the final configuration of the four agents with the circle followed by the leader.

The desired distance between agents is now $1.3 \mathrm{~m}$. The path followed by the leader is unchanged. A set of 4 electrodes (corresponding to line 5 of table I) is considered. We compare the results obtained when the outputs are designed for a distance of $1.8 \mathrm{~m}$ or $1.3 \mathrm{~m}$, qualitatively in Figure 7 and quantitatively in table II. The proposed control law is enough robust to be used with stability for several desired distances between agents, but the performance in term of path followed by the agents is disturbed when the outputs are inappropriately chosen.

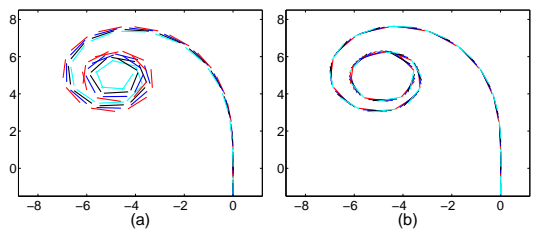

Fig. 7. The path followed by 4 agents with 4 electrodes and a desired distance between agents is $1.3 \mathrm{~m}$. The output are designed for a distance between agents of $1.8 \mathrm{~m}$ in (a), or $1.3 \mathrm{~m}$ in (b).

\footnotetext{
${ }^{1}$ limited to the part where the closest point of the path of the leader centre is not the fist point to avoid the beginning of the motion where the leader path is not defined.
}

\begin{tabular}{|c|c|c|c|c|c|}
\hline & \multicolumn{3}{|c|}{$d=1.8 \mathrm{~m}$} & \multicolumn{3}{|c|}{$d=1.3 \mathrm{~m}$} \\
& 2 elec & $3 \mathrm{elec}$ & 4 elec & $d=1.3$ & $d=1.8$ \\
\hline \hline agent 1 & 0.1426 & 0.0279 & 0.0196 & 0.0083 & 0.1143 \\
\hline agent 2 & 0.2479 & 0.0571 & 0.0407 & 0.0168 & 0.2220 \\
\hline agent 3 & 0.2746 & 0.0868 & 0.0639 & 0.0257 & 0.3151 \\
\hline
\end{tabular}

TABLE II

AVERAge Distance BETWEEN THE PATH OF THE LEADER AND OF THE $i^{t h}$ AGENT IN $m$ FOR AN AGENT OF $1 m$.

\section{CONCLUSION}

By combining rules inspired by electric fish with a follower-leader multi-agent strategy, we have addressed the problem of navigating while maintaining a file formation of a group of non-holonomic agents. The control strategy is based on the direct servo control of the electric measurements. We have defined a preferable behaviour for the file formation with curved path of the leader. A tracking of the path of the leader is expected rather that a strict alignment of agents in order to reduce the swept space by the formation. A methodology has been proposed to choose the controlled outputs that lead to the desired behaviour. The efficiency of the methodology has been shown in simulation. The importance of the number and position of the sensor on the performance of the control has also been illustrated.

\section{REFERENCES}

[1] N. Servagent, B. Jawad, S. Bouvier, F. Boyer, A. Girin, F. Gomez, V. Lebastard, C. Stefanini, and P.-B. Gossiaux, "Bioinspired sensor for electrolocation and navigation in conducting media," IEEE SENSORS JOURNAL, accepted, 2013.

[2] H. Lissmann and K. Machin, "The mechanism of object location in gymnarchus niloticus and similar fish," The Journal of Experimental Biology, no. 35, pp. 451-486, 1958.

[3] C. Hopkins, "Electrical perception and communication," in Encyclopedia of Neuroscience, in: squire lr ed. New York: Oxford: Academic Press, 2009, vol. 3, pp. 813-831.

[4] M. E. Arnegard and B. Carlson, "Electric organ discharge patterns during group hunting by a mormyrid fish," Proc. Royal Society Biological sciences, vol. 272, no. 1570, pp. 1305-1314, 2005.

[5] Y. Morel, M. Porez, and A. Ijspeert, "Estimation of the relative position and coordination of mobile underwater robotic platforms through electric sensing," in ICRA, 2012.

[6] V. Lebastard, C. Chevallereau, A. Amrouche, B. Jawad, A. Girin, F. Boyer, and P. Gossiaux, "Underwater robot navigation around a sphere using electrolocation sense and kalman filter," in IROS, 2010.

[7] C. Samson, M. Le Borgne, and B. Espiau, Robot Control: the Task Function Approach. Oxford Science Publications, 1991.

[8] B. Espiau, F. Chaumette, and P. Rives, "A new approach to visual servoing in robotics," IEEE Trans. on Robotics and Automation, vol. 8 , no. 3, pp. 313-326, 1992.

[9] F. Chaumette and S. Hutchinson, "Visual servo control, part i: Basic approaches," IEEE Robotics and Automation Magazine, vol. 4, no. 13, pp. 82-90, 2006.

[10] C. Chevallereau, F. Boyer, V. Lebastard, and M. Benachenhou, "Electric sensor based control for underwater multi-agents navigation in formation," in ICRA, 2012.

[11] N. E. Leonard and E. Fiorelli, "Virtual leaders, artificial potentials and coordinated control of groups," in IEEE Conference on Decision and Control, 2001, pp. 2968-2973.

[12] F. Boyer, P. Gossiaux, B. Jawad, V. Lebastard, and M.Porez., "Model for a sensor bio-inspired from electric fish," IEEE transactions on robotics, vol. 28, no. 2, pp. $492-505,2012$.

[13] A. Isidori, Nonlinear Control Systems. 3rd ed. Berlin: SpringerVerlag, 1995. 